\title{
Cáncer de vesícula biliar como hallazgo histopatológico posterior a colecistectomía. Prevalencia e incidencia a trece años en el Hospital Angeles Pedregal
}

\author{
Gallbladder cancer as a histopathological finding after cholecystectomy. \\ Prevalence and incidence at thirteen years in Hospital Angeles Pedregal
}

\author{
Edna M Aizpuru, * Tania Karina Cázares Robles, * José Jesús Castillo García,* Guillermo León Merino,* \\ Adriana Cecilia Gallegos Garza, ${ }^{\ddagger}$ Ingrid Flandes Aguilar, $\$$ Emmanuel De Icaza Murua \\ Citar como: Aizpuru EM, Cázares RTK, Castillo GJJ, León MG, Gallegos GAC, Flandes Al et al. Cáncer de vesícula \\ biliar como hallazgo histopatológico posterior a colecistectomía. Prevalencia e incidencia a trece años en el \\ Hospital Angeles Pedregal. Acta Med GA. 2022; 20 (1): 37-42. https://dx.doi.org/10.35366/103554
}

\section{Resumen}

Introducción: El carcinoma de vesícula biliar (CVB), descrito por primera vez en 1777 , ocupa el sexto lugar en frecuencia de neoplasias malignas en el tubo digestivo. Objetivo: Conocer la prevalencia e incidencia del cáncer de vesícula biliar en pacientes postoperados de colecistectomía en el Hospital Angeles Pedregal. Material y métodos: Estudio descriptivo, observacional, retrospectivo y transversal de los expedientes clínicos de los pacientes sometidos a colecistectomía, incluyendo 6,954 colecistectomías durante 13 años. Se analizaron los resultados del estudio histopatológico, se recolectaron para obtener la prevalencia e incidencia del CVB. Resultados: De los 6,954 pacientes estudiados, se diagnosticaron 16 con CVB (0.23\%), presentando una prevalencia de 0.23 casos por cada 100 colecistectomías, e incidencia de 2.3 por cada 1,000 casos sometidos a este procedimiento. El tipo histológico más común fue adenocarcinoma con 15 casos y uno de carcinoma neuroendocrino. Sólo en dos de los 16 casos (12.5\%) se sospechó CVB en forma preoperatoria. Conclusiones: Los porcentajes obtenidos de CVB en nuestro hospital están por debajo de los límites reportados en la literatura internacional. De igual manera, la sospecha de porcentaje de CVB está por debajo de lo reportado en estudios de otras poblaciones. La combinación de ultrasonido y tomografía pueden aumentar el porcentaje de sospecha de CVB de forma preoperatoria.

Palabras clave: Cáncer de vesícula biliar, cáncer de vía biliar, incidencia, prevalencia.

\section{Abstract}

Introduction: Gallbladder carcinoma (GC), first described in 1777 , ranks sixth in the frequency of malignancies in the gastrointestinal tract. Objective: To determine the prevalence and incidence of GC in patients after cholecystectomy at Hospital Angeles Pedregal. Material and methods: We conducted a descriptive, observational, retrospective, and cross-sectional study of clinical records of patients undergoing cholecystectomy, including a total of 6,954 cholecystectomies for thirteen years. Histological reports were collected and analyzed to obtain GC prevalence and incidence. Results: Sixteen patients of the 6,954 studied cases were diagnosed with $\mathrm{GC}(0.23 \%)$, with a prevalence of 0.23 cases per 100 cholecystectomies and with an incidence of 2.3 for every 1,000 subjects. Adenocarcinoma was the most common histopathological diagnosis with 15 cases, and only one neuroendocrine tumor. Preoperative GC diagnosis was suspected in only two of the 16 patients with malignancy (12.5\%). Conclusions: The incidence of GC in our hospital is located below the lower limits reported in the literature. Preoperative suspicion is also under the rates estimated worldwide; therefore, ultrasound and computerized tomography can raise the percentage of preoperatively GC detection.

Keywords: Gallbladder cancer, bile duct cancer, incidence, prevalence.
* Cirugía General, Hospital Angeles Pedregal. Facultad Mexicana de Medicina de la Universidad La Salle de México. Ciudad de México, México.

₹ Servicio de Anatomía Patológica, Hospital Angeles Pedregal. Ciudad de México, México.

§ Residente de Cirugía General, Universidad Nacional Autónoma de México. Hospital Central Sur de Alta Especialidad PEMEX. Ciudad de México, México.

^ Residente de Oftalmología, Instituto Mexicano de Oftalmología. Querétaro, México.
Correspondencia:

Tania Karina Cázares Robles

Correo electrónico: ae.crazares@gmail.com

Aceptado: 14-06-2021.

www.medigraphic.com/actamedica 


\section{INTRODUCCIÓN}

El cáncer de vesícula biliar (CVB) es la neoplasia biliar extrahepática más común. Ocupa el sexto lugar en frecuencia de neoplasias malignas en el tubo digestivo en Estados Unidos. ${ }^{1}$ Fue descrito hace más de dos siglos por Maximilian Stoll, en donde se comenta por primera vez como una patología maligna y poco frecuente, desde entonces múltiples estudios han establecido un patrón característico de diagnóstico tardío y tratamiento ineficaz en esta patología. ${ }^{2}$

Se ha descrito la cirugía como única terapia que brinda la mejor posibilidad de curación y mejora la supervivencia; sin embargo, ésta requiere una resección quirúrgica adecuada, se recomienda la resección hepática de los segmentos IV B y $\vee$ para la obtención de márgenes negativos acompañada de linfadenectomía. En etapas tempranas, se ha reportado una tasa de supervivencia a cinco años de $75.7-94.7 \%$. Por lo tanto, la extirpación completa del tumor debe ser el principal objetivo del tratamiento para el CVB resecable. ${ }^{1}$

Los factores de riesgo reportados son edad mayor a 65 años, sexo femenino, la inflamación crónica, colelitiasis, pólipos (> $1 \mathrm{~cm})$, anomalías en la unión pancreatobiliar, entre otros menos comunes. ${ }^{3,4}$ Asimismo, se consideran factores predisponentes el síndrome de Mirizzi, enfermedad por reflujo gastroesofágico y calcificación de la pared vesicular. ${ }^{5}$

Los pólipos de la vesícula biliar se observan en hasta $12 \%$ de las muestras de colecistectomía y entre 5 y $7 \%$ de los estudios de ecografía biliar, pero sólo $0.6 \%$ de los pólipos son malignos. ${ }^{6}$

Afecta al sexo femenino dos a tres veces más que al masculino, varía en diferentes partes del mundo, principalmente en regiones de alta prevalencia. El estrógeno está relacionado como causa en el aumento de la sobresaturación del colesterol en la bilis y, por lo tanto, participa en la patogénesis mediada por cálculos biliares. ${ }^{5}$

Se ha reportado un amplio rango de prevalencia entre 2-27.3/100,000 habitantes, siendo los habitantes de Chile, India, Polonia, Pakistán y los indios americanos los que más alta incidencia tienen, encontrándose las tasas más bajas en Europa. ${ }^{7}$

No existen estadísticas precisas, aunque en México en 2002 se reportaron histológicamente 1,022 casos de CVB, lo que nos ubica en el décimo primer lugar por incidencia (0.7 por 100,000 habitantes/año). ${ }^{8}$

Existe una relación inversamente proporcional entre el número de colecistectomías realizadas con respecto a la incidencia de CVB, ya que ésta es extraída antes de que presente cambios displásicos o carcinoma, sumando así importancia a la prevención de factores de riesgo como infección crónica y colelitiasis de larga evolución. Las tasas de cáncer vesicular incidental (diagnosticado durante el acto quirúrgico o por reporte de patología postoperatorio) varía entre regiones, reportándose en diferentes series tasas de entre 0.2 y $3.3 \%{ }^{3}$ Aproximadamente uno de cada 250 colecistectomías laparoscópicas producirá un diagnóstico patológico de CVB incidental. ${ }^{6}$

La variedad histológica más común del cáncer de vesícula es el adenocarcinoma, aunque se han reportado tumores neuroendocrinos, tumores de células pequeñas, linfoma primario no Hodgkin, así como metástasis. ${ }^{6}$

Todos los subtipos de cáncer biliar son raros y tienen mal pronóstico. El pronóstico depende del estadio en que se encuentra el tumor, de acuerdo con la clasificación de la American Joint Committee on Cancer (AJCC) basada en la profundidad de invasión hacia la pared vesicular y su extensión hacia órganos y ganglios linfáticos adyacentes. ${ }^{9}$ La supervivencia general a cinco años es de $50 \%$ para el cáncer de vesícula en etapa I y de 3\% para una etapa IV. ${ }^{6}$

Existen múltiples sistemas de estadificación para el CVB. En esta investigación se muestra la clasificación TNM según la AJCC de cáncer vesicular (Tabla 1).9

El cuadro clínico es difícil de diferenciar de una patología benigna, ya que se presenta en la mayoría como cólico biliar, asociado con pérdida de peso e ictericia, ésta última es una contraindicación para la resección, ya que se ha visto que aun realizando resección R0 se observa una supervivencia de seis meses. ${ }^{6}$

El diagnóstico por imagen puede observarse como una masa adyacente a la vesícula biliar, pólipos o un engrosamiento en la pared. Sin embargo, el engrosamiento de la pared puede ser un indicativo de enfermedad benigna. Los hallazgos que favorecen son: discontinuidad o engrosamiento irregular de la mucosa, serosa o pérdida de la diferenciación entre estas capas. Cualquier tumor descubierto por patología o ecografía debe someterse a tomografía computarizada (TC) de tórax, abdomen y pelvis, para la identificación de implantes peritoneales, invasión vascular, y así poder determinar su resecabilidad con una sensibilidad de $99 \%$ y especificidad de $76 \%$. La resonancia magnética con gadolinio es utilizada para exploración de conductos biliares y parénquima hepático. ${ }^{6}$

El CVB es el cáncer del tracto biliar con la menor mediana de supervivencia. De acuerdo con la Sociedad Americana de Cáncer, uno de cada cinco se diagnosticará cuando aún permanezca localizado sólo en la vesícula biliar. La mayoría será diagnosticado cuando el tumor se ha extendido fuera de la vesícula biliar, lo que limita drásticamente las opciones disponibles para el tratamiento curativo y reduce la supervivencia general. ${ }^{10}$

El mal pronóstico de los pacientes con diagnóstico de CVB conlleva a la necesidad de mejorar las estrategias para integrar el conocimiento de los factores asociados con el 
Tabla 1: Clasificación TNM según la AJCC (American Joint Committee on Cancer) de cáncer vesicular.

\begin{tabular}{|c|c|c|c|c|}
\hline Estadio & & $\mathrm{T}$ (tumor) & N (nódulos) & M (metástasis) \\
\hline 0 & & Carcinoma in situ & - & - \\
\hline \multirow[t]{2}{*}{1} & $\mathrm{~T} 1 \mathrm{a}$ & Tumor que invade la lámina propia & - & - \\
\hline & $\mathrm{T} 1 \mathrm{~b}$ & Tumor que invade la capa muscular & - & - \\
\hline$\| A$ & $\mathrm{~T} 2 \mathrm{a}$ & $\begin{array}{l}\text { Tumor que invade el tejido conectivo } \\
\text { perimuscular sin extenderse más allá de la } \\
\text { serosa }\end{array}$ & & \\
\hline IIB & $\mathrm{T} 2 \mathrm{~b}$ & $\begin{array}{l}\text { Tumor que invade el tejido conectivo } \\
\text { perimuscular sin extenderse más allá del } \\
\text { hígado }\end{array}$ & - & - \\
\hline IIIA & T3 & $\begin{array}{l}\text { Tumor que perfora la serosa o invade } \\
\text { directamente hacia el hígado o un órgano } \\
\text { adyacente }\end{array}$ & - & - \\
\hline IIIB & T1-3 & $\begin{array}{l}\text { Tumor que puede o no invadir el hígado o } \\
\text { estructuras adyacentes, pero no ha crecido } \\
\text { hacia la vasculatura hepática (portal o de arteria } \\
\text { hepática) }\end{array}$ & $\begin{array}{l}\text { Con extensión a no más de } 3 \text { ganglios } \\
\text { linfáticos adyacentes }\end{array}$ & - \\
\hline IVA & $\mathrm{T} 4$ & $\begin{array}{l}\text { Tumor que se extiende hacia la vasculatura } \\
\text { hepática (portal o de arteria hepática) o a } 20 \\
\text { más órganos fuera del hígado }\end{array}$ & $\begin{array}{l}\text { Con o sin extensión a no más de } 3 \\
\text { ganglios linfáticos adyacentes }\end{array}$ & - \\
\hline \multirow[t]{2}{*}{ IVB } & T1-4 & $\begin{array}{l}\text { El tumor puede extenderse o no fuera de la } \\
\text { vesícula }\end{array}$ & $\begin{array}{l}\text { Con extensión a } 4 \text { o más ganglios } \\
\text { linfáticos adyacentes }\end{array}$ & - \\
\hline & T1-4 & $\begin{array}{l}\text { El tumor puede extenderse o no fuera de la } \\
\text { vesícula }\end{array}$ & $\begin{array}{l}\text { Con o sin extensión a ganglios } \\
\text { linfáticos adyacentes }\end{array}$ & $\begin{array}{l}\text { Metástasis a } \\
\text { distancia }\end{array}$ \\
\hline
\end{tabular}

desarrollo del cáncer de vesícula para promover programas que aceleren la detección y prevención del cáncer de vesícula biliar en etapas más tempranas.

Objetivo. Conocer la prevalencia e incidencia del cáncer de vesícula biliar en pacientes postoperados de colecistectomía en el Hospital Angeles Pedregal.

\section{MATERIAL Y MÉTODOS}

Se realizó un estudio retrospectivo, descriptivo y observacional de los expedientes clínicos de los pacientes sometidos a cirugía de colecistectomía por cualquier patología de origen biliar. El método de búsqueda fue a través del archivo del Departamento de Patología del Hospital Angeles Pedregal, así como de los expedientes pertenecientes al archivo clínico del mismo hospital. Los criterios tomados en cuenta para ser incluidos en el estudio fueron todos los casos sometidos a cirugía de colecistectomía por cualquier patología de vesícula biliar (cuya razón primaria fuera la colecistectomía) realizada entre el $1^{\circ}$ de enero de 2004 y el 31 de octubre de 2017. Se excluyeron aquéllos cuya indicación de colecistectomía hubiera sido causa secundaria al procedimiento quirúrgico, así como expedientes de pacientes intervenidos quirúrgicamente en otro hospital. De los expedientes clínicos con cáncer de vesícula biliar confirmado por reporte de histopatología, se recopilaron los siguientes antecedentes: edad, sexo, diagnóstico clínico previo a la intervención quirúrgica y manifestaciones clínicas preoperatorias. Del archivo de anatomía patológica se obtuvo el tipo histológico, grado de diferenciación celular y el grado de infiltración a la pared de la vesícula biliar o a órganos vecinos (estadificación de Nevin). Con fines de análisis estadístico, se calculó y analizó la prevalencia y la incidencia en la población del Hospital Angeles Pedregal.

\section{RESULTADOS}

Los resultados del presente estudio determinaron un total de 6,954 pacientes operados de colecistectomía entre el $1^{\circ}$ de enero de 2004 y el 31 de octubre de 2017, de éstos, 4,523 (65.04\%) fueron del sexo femenino y 2,431 (34.96\%) masculinos, lo que da una relación de 2:1, con un promedio de $25.9 \%$ entre los 30 y 39 años en ambos sexos (Figura 1).

En cuanto a la frecuencia de cáncer de vesícula biliar, se encontraron 16 casos con reporte histopatológico confirmatorio. En la distribución de presentación por grupos 
Figura 1: Frecuencia de colecistectomías por grupo de edad.

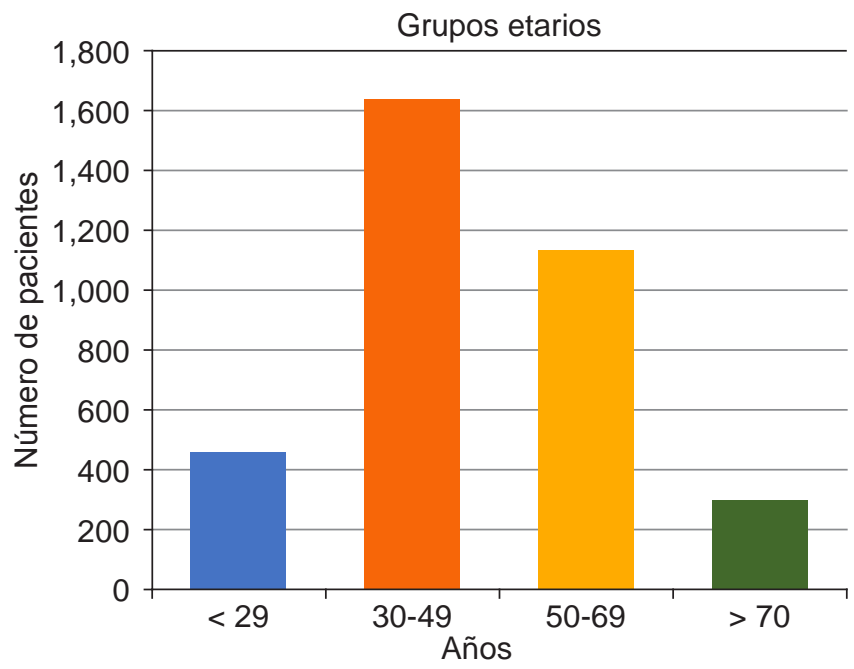

de edad, se encontró una mayor frecuencia en el grupo de 50 a 69 años con nueve casos, uno en cada grupo etario de 30 a 49 años y seis casos en el grupo mayor de 70 años.

La prevalencia de CVB fue de 0.23 casos por cada 100 sometidos a cirugía de colecistectomía como indicación quirúrgica primaria, en tanto la incidencia fue de 2.3 por cada 1,000 sometidos a colecistectomía. El CVB se presentó con mayor frecuencia en mujeres que en hombres a razón de 3:1 (12 mujeres y cuatro hombres).

Hallazgos anatomopatológicos de los 16 pacientes con diagnóstico de CVB, se reportó adenocarcinoma en 15 y carcinoma neuroendocrino en uno. De acuerdo con el grado de diferenciación celular, se encontró que cuatro se presentaban con cáncer bien diferenciado (25\%), ocho $(50 \%)$ presentaron moderado grado de diferenciación celular, y cuatro presentaron adenocarcinoma poco diferenciado (25\%).

De los 16 pacientes con diagnóstico anatomopatológico de cáncer, se encontró que de acuerdo con el grado de invasión tumoral, en ninguno se presentó tumor que sólo infiltrara la mucosa (T1b); en cuatro (25\%) el tumor infiltraba la capa muscular (T2), en seis $(37.5 \%)$ el tumor infiltraba de forma transmural la pared vesicular $\leq 2 \mathrm{~cm}$ a lecho hepático, de los cuales un paciente $(6.2 \%)$ tenía afectación del ganglio cístico (T3), y en seis el tumor infiltraba el lecho hepático $>2$ cm y/o presentaban metástasis a otros órganos (37.5\%) (T4) (Tabla 1).

Los hallazgos clínicos encontrados en los 16 casos con CVB fueron dolor en cuadrante superior derecho en 11 $(68.7 \%)$, ictericia en seis $(37.5 \%)$, anorexia o hiporexia en seis $(37.5 \%$ y y pérdida de peso en tres (18.7\%). El diagnóstico preoperatorio de los casos de cáncer vesicular se realizó a través del cuadro clínico y de estudios de gabinete, encontrando la siguiente relación: siete pacientes $(43.7 \%)$ con colecistitis crónica litiásica, dos con datos ultrasonográficos compatibles con piocolecisto (12.5\%), tres con colecistitis crónica litiásica no agudizada (18.7\%), dos con diagnóstico de coledocolitiasis $(12.5 \%)$ y dos pacientes con datos tomográficos compatibles con cáncer vesicular (12.5\%).

\section{DISCUSIÓN}

El número de personas sometidas a colecistectomía durante el periodo mencionado es similar al descrito en otros estudios internacionales en un periodo similar (2004-2019) con 6,314 casos. $^{3}$

En una publicación mexicana longitudinal, retrospectiva, observacional a cinco años (2009-2013) en donde se obtuvo un total de 903 colecistectomías, se encontró una prevalencia de $0.4 \%$, siendo la colecistitis crónica y aguda los factores asociados más frecuentes. ${ }^{11}$

Es importante mencionar los resultados obtenidos durante un periodo de seis años en la misma población, en donde se reportó una prevalencia de 0.26 , lo cual podría ser un indicador de mantenimiento de una población estable. ${ }^{12}$

Encontramos que la prevalencia de CVB en nuestra población fue 0.23 casos por cada 100 pacientes sometidos a colecistectomía, con indicación primaria del procedimiento quirúrgico que, en comparación con otras series, está por debajo de lo reportado en estadísticas mundiales, las cuales van entre 2.1 y $8.8 \%,{ }^{10-12}$ y algunas otras series reportan entre 1 y $2.8 \%$. $^{13,14}$

Sin embargo, en los hallazgos de esta investigación ningún paciente presentó tumores in situ o circunscritos a

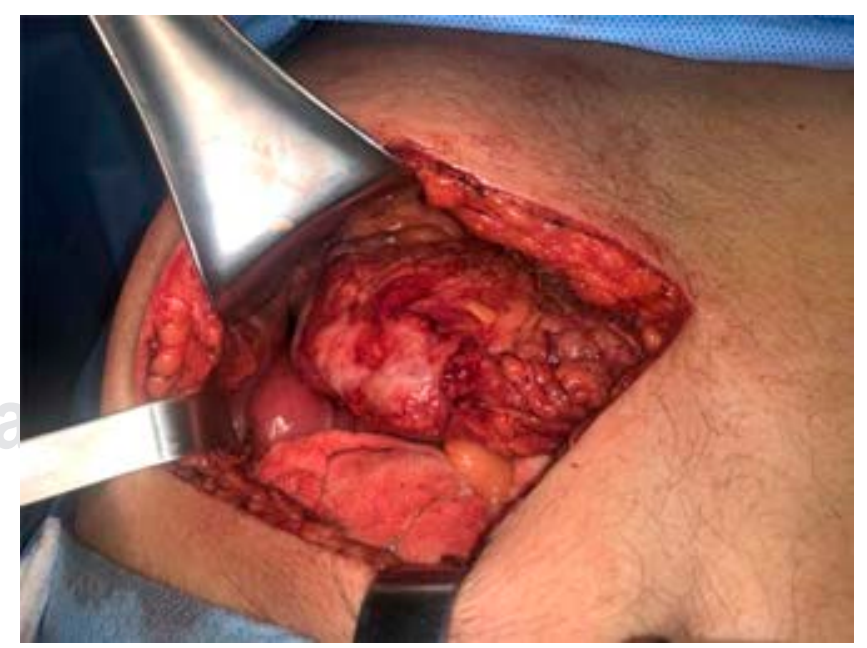

Figura 2: Adenocarcinoma de vesícula biliar infiltrando lecho hepático. 
la pared muscular (T1) y más de una tercera parte de los casos de cáncer vesicular se encontraban con extensión tumoral hacia lecho hepático $>2 \mathrm{~cm}$ o a órganos adyacentes (T4), mientras que otros estudios internacionales han reportado hallazgos patológicos con tumores $\mathrm{T} 1 \mathrm{~b}$ en 14 casos (10.3\%), T2 en 68 (50.0\%), T3 en 54 (39.7\%), y ningún caso con tumores T4. ${ }^{15}$ Esto podría explicarse por el retraso en el diagnóstico de colelitiasis o pólipo en nuestro país, siendo los factores de riesgo para cáncer vesicular más importantes en asintomáticos, lo que permite la progresión de la enfermedad hasta etapas más avanzadas.

De los casos con cáncer de vesícula biliar, encontramos que el tipo histológico más frecuente fue el adenocarcinoma (15 de 16), es decir, 93.7\%, y sólo un caso de tumor neuroendocrino, lo que es parcialmente similar a lo reportado en la literatura mundial, ya que el segundo tipo de cáncer de vesícula más común es el escamoso, seguido del adenoescamoso (Figura 2). ${ }^{16-19}$

De acuerdo al grado de diferenciación celular, encontramos que una cuarta parte de los casos se reportaron como cáncer bien diferenciado, y la mitad con cáncer moderadamente diferenciado, que coincide con el mayor porcentaje encontrado de tumores moderadamente diferenciados en otros estudios internacionales; sin embargo, en este estudio se encontró que una tercera parte de los pacientes presentaban cáncer poco diferenciado, lo cual no concuerda con los hallazgos en otras series. ${ }^{20}$

Esto junto con el grado de infiltración tumoral, son factores que influyen en la supervivencia a cinco años en los casos con cáncer de vesícula biliar. La AJCC creó la clasificación por TNM, que es la base para la estadificación del CVB y a la vez para su tratamiento. ${ }^{9}$

Con lo anterior, ante la sospecha de cáncer vesicular preoperatorio, es necesario realizar estudios de imagen para planear mejor el tipo de abordaje quirúrgico con intención curativa, realizando en la mayoría de las veces un procedimiento radical en un sólo tiempo; por otro lado, la detección intraoperatoria de cáncer vesicular durante el procedimiento laparoscópico deberá ser convertido a cirugía abierta para poder ofrecer de igual manera un tratamiento definitivo. ${ }^{9}$

En los casos de diagnóstico postoperatorio de cáncer vesicular, tras obtener el reporte de patología, la reintervención para poder resecar los márgenes positivos deberá incluir la congelación de un corte intraoperatorio del muñón cístico, cuya negatividad excluye la necesidad de resecar la vía biliar con propósitos oncológicos. ${ }^{9}$

\section{CONCLUSIONES}

A pesar de que la incidencia de cáncer vesicular entre colecistectomizados es baja, el beneficio de la detección oportuna de malignidad en los reportes de patología de rutina continúa siendo una estrategia costoefectiva. De forma alternativa, puede proponerse el tamizaje individual de casos con respecto a los factores de riesgo, hallazgos intraoperatorios y la examinación del espécimen de patología con la finalidad de determinar el grado de diferenciación celular, invasión tumoral y etapificación clínica, estableciendo así el tratamiento, ya sea quirúrgico o no, para cada caso.?

El bajo porcentaje de pacientes con sospecha preoperatoria de CVB quizá tiene relación directa con los estudios radiológicos que se realizaron antes de ser sometidos a cirugía, ya que, según lo reportado en la literatura, el aumento en el porcentaje de diagnóstico preoperatorio de cáncer vesicular se debe a la combinación de estudios de ultrasonografía y tomografía, los cuales fueron realizados de esta forma en sólo dos pacientes, en los que se sospechó el diagnóstico de malignidad de forma preoperatoria.

\section{REFERENCIAS}

1. Maegawa FB, Ashouri Y, Hamidi M, Hsu CH, Riall TS. Gallbladder cancer surgery in the United States: lymphadenectomy trends and impact on survival. J Surg Res [Internet]. 2021; 258: 54-63. Available in: https://doi.org/10.1016/j.jss.2020.08.041

2. Andrén-Sandberg A, Deng Y. Aspects on gallbladder cancer in 2014. Curr Opin Gastroenterol. 2014; 30 (3): 326-331.

3. Kanlioz M, Ekici U, Ayva Y. Analysis of incidental gallbladder cancer in cholecystectomies. Cureus. 2019; 11 (9): e5710.

4. Muszynska C, Nilsson J, Lundgren L, Lindell G, Andersson R, Sandstrom $P$ et al. A risk score model to predict incidental gallbladder cancer in patients scheduled for cholecystectomy. Am J Surg. 2020; 220 (3): 741-744.

5. Sharma A, Sharma KL, Gupta A, Yadav A, Kumar A. Gallbladder cancer epidemiology, pathogenesis and molecular genetics: Recent update. World J Gastroenterol. 2017; 23 (22): 3978-3998.

6. Hickman L, Contreras C. Gallbladder cancer: diagnosis, surgical management, and adjuvant therapies. Surg Clin North Am [Internet]. 2019; 99 (2): 337-355. Available in: https://doi.org/10.1016/j. suc. 2018.12.008

7. Wrenn SM, Callas PW, Abu-Jaish W. Histopathological examination of specimen following cholecystectomy: Are we accepting resect and discard? Surg Endosc. 2017; 31 (2): 586-593.

8. Chan NC, Bandín MA, Villalobos BI, Torres MA. Guía de práctica clínica. Cáncer de vesícula biliar. México: Asociación Mexicana de Cirugía General; 2014.

9. Edition S. AJCC Cancer Staging Manual. AJCC Cancer Staging Manual. 2017.

0. Schmidt MA, Marcano-Bonilla L, Roberts LR. Gallbladder cancer: epidemiology and genetic risk associations. Chin Clin Oncol. 2019; 8 (4): 31.

11. Noriega UVM, Álvarez SM, Hernández MR, Rodríguez LR, Bieletto TEO, Fenig RJ. Carcinoma de vesícula biliar incidental después de colecistectomía. Análisis de los hallazgos patológicos a cinco años. Acta Med. 2016; 14 (4): 196-200.

12. Castillo GJJ, Romo AC, Ruiz LJ, Escrivá MJF, Córdova PVH. Cáncer de vesícula biliar como hallazgo histopatológico posterior a la colecistectomía. Prevalencia e incidencia en el Hospital Ángeles Pedregal. Acta Med. 2010; 8 (3): 140-147. 
13. Aristi UG, Pérez-Peña Díaz-Conti M, Soto AMV. Carcinoma de vesícula biliar: una neoplasia subestimada. Rev Med Hosp Gen Mex. 1999; 62 (2): 94-101.

14. Ramírez CP, Suárez MA, Santoyo J, Fernández JL, Jiménez M, Pérez JA et al. Actualización del diagnóstico y el tratamiento del cáncer de vesícula biliar. Cir Esp. 2002; 71: 102-111.

15. Butte JM, Gonen M, Allen PJ, D'Angelica MI, Kingham TP, Fong $Y$ et al. The role of laparoscopic staging in patients with incidental gallbladder cancer. HPB (Oxford). 2011; 13 (7): 463-472.

16. Levy AD, Murakata LA, Rohrmann CA Jr. Gallbladder carcinoma: radiologic-pathologic correlation. Radiographics. 2001; 21 (2): 295-314.
17. Bhuta P, Brown MG, Alderdice JM. Gall bladder cancer--radical surgery, the key role to improve outcome. Ulster Med J. 2002; 71 (2): 128-131.

18. Reid KM, Ramos-De la Medina A, Donohue JH. Diagnosis and surgical management of gallbladder cancer: a review. J Gastrointest Surg. 2007; 11 (5): 671-681.

19. Mghirbi F, Ayadi M, Karray W, Yahyaoui Y, Meddeb K, Mokrani A et al. Squamous cell carcinoma of the gallbladder. Trans/ Gastroenterol Hepatol. 2016; 1: 78.

20. Regimbeau JM, Fuks D, Bachellier P, Le Treut YP, Pruvot FR, Navarro F et al. Prognostic value of jaundice in patients with gallbladder cancer by the AFC-GBC-2009 study group. Eur J Surg Oncol [Internet]. 2011; 37 (6): 505-512. Available in: http://dx.doi.org/10.1016/j.ejso.2011.03.135 